\title{
Maternal and Perinatal Outcome in Hypertensive Disorders in Pregnancy
}

\author{
Sapna IS ${ }^{1 *}$ and Saroja ${ }^{2}$ \\ ${ }^{1}$ Professor, Dept of OBG, SSIMS\& RC, Davangere, Karnataka, India \\ ${ }^{2}$ Postgraduate, Dept of OBG, SSIMS\& RC, Davangere, Karnataka, India
}

Received: 眥 April 23, 2018; Published: 制 May 01, 2018

*Corresponding author: Dr Sapna IS, Professor, Dept of OBG, SSIMS\& RC, Davangere, Karnataka, India, Email: sreddyabhi@gmail.com

\section{Abstract}

Hypertensive disorders of pregnancy still holds major cause of maternal and perinatal morbidity and mortality \& the commonest cause of referral to a tertiary centre.

Objective: study the maternal and foetal outcome in patient with hypertensive disorder in a tertiary centre from june2016 to may2017 (one year).

Method: Total number of women with severe preeclampsia and eclampsia after 20 wks were 146 . Women with medical complications were excluded. Hypertensive women were managed as per existing protocol of the institution, after detailed history, examination and investigations. Anti-hypertensive of choice was labetalol and oral nefedipine, Mgso4 was used as anti-convulsant.

Result: Out of 146 cases of severe pre-eclampsia and eclampsia, majority 91(62.32\%) were between 21-25yrs years of age and 76(52\%) were primigravida. We had 21(14.38\%) patients with h/o convulsions and 103(70.5\%) with severe pre-eclampsia of whom $3(2.9 \%)$ had convulsions. Headache was most common complaint. Common mode of delivery was caesarean section $106(72.6 \%)$ women, majority in view of failed induction or non-progress. Maternal complications were noted 43(29.5\%) attributed to renal dysfunction, postpartum haemorrhage, DIC, placental abruption, HELLP, pulmonary oedema Total 146 cases ,live birth 119 (81\%), 24\% were required NICU care, 15(10.27\%) cases were IUFD, 4(2.73\%) babies stillborn.2 (1.36\%) neonatal deaths,1(0.68\%) perinatal death.

Conclusion: Maternal and perinatal complications are more in patients with eclampsia. The incidence of eclampsia can be reduced by regular antenatal care, early recognition and prompt treatment of severe pre-eclampsia.

Background: Hypertensive disorders of pregnancy accounts for the majority of referrals in a tertiary centre as it stands one of the major causes of maternal and perinatal morbidity and mortality. The objective was to study the maternal and foetal outcome in patient with hypertensive disorder in a tertiary centre over a period of one year.

\section{Introduction}

Hypertension in pregnancy constitutes the most common medical complication occurring in $12-22 \%$ of all pregnancies of which pre-eclampsia remains the leading cause that complicates $10 \%$ of all pregnancies. It is defined as new onset of elevated blood pressure and proteinuria $(\mathrm{BP}>/=140 / 90$ and $>/=0.3 \mathrm{gm}$ protein in 24 hours urine specimen) after 20 weeks of gestation in a previously normotensive woman. It is considered severe if blood pressure and proteinuria are increased substantially $(B P>/=160 / 110$ and $>5$ gm protein in 24 hours urine specimen) or symptoms of end organ damage are present. Eclampsia is defined as the new onset of generalized tonic clonic seizure in a woman with severe preeclampsia. WHO estimated that approximately 60,000 women die each year from pre-eclampsia world-wide Pre-eclampsia and eclampsia account for $24 \%$ of all [1-3]. Maternal deaths in India, mainly attributed to complications of preeclampsia and eclampsia 


\section{Method}

Total 146 women with severe preeclampsia and eclampsia after 20 wks were included. Women with medical complications were excluded [4,5]. Patients were managed as per existing protocol after proper history, examination and investigations. Anti-hypertensive of choice was labetalol and oral nefedipine, Mgso4 was used as anti convulsant.

\section{Result}

Out of 146 cases of severe pre-eclampsia and eclampsia, majority 91(62.32\%) were between 21-25yrs years of age and $76(52 \%)$ were primigravida. We had 21(14.38\%) patients with h/o convulsions and $103(70.5 \%)$ with severe pre-eclampsia of whom $3(2.9 \%)$ had convulsions. Headache was most common complaint. Common mode of delivery was caesarean section 106(72.6\%) women, majority in view of failed induction or non-progress [68]. Maternal complications were noted in 43(29.5\%) attributed to renal dysfunction, postpartum haemorrhage, DIC, placental abruption, HELLP, pulmonary oedema (Tables 1-3).
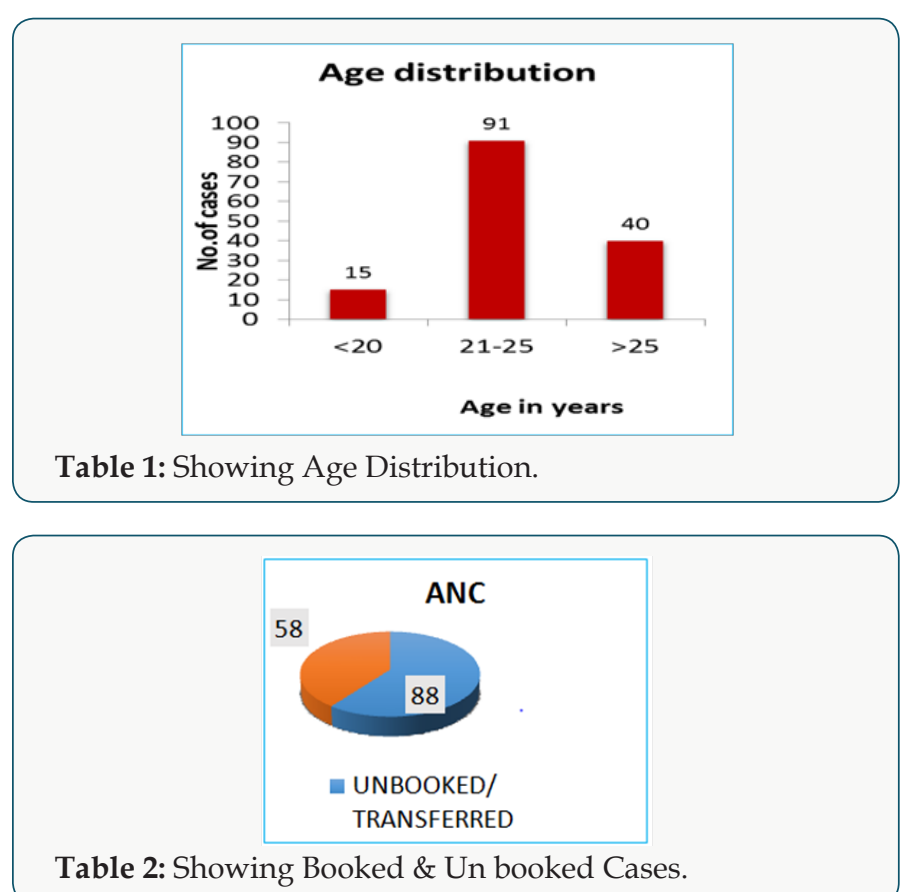

Table 2: Showing Booked \& Un booked Cases.

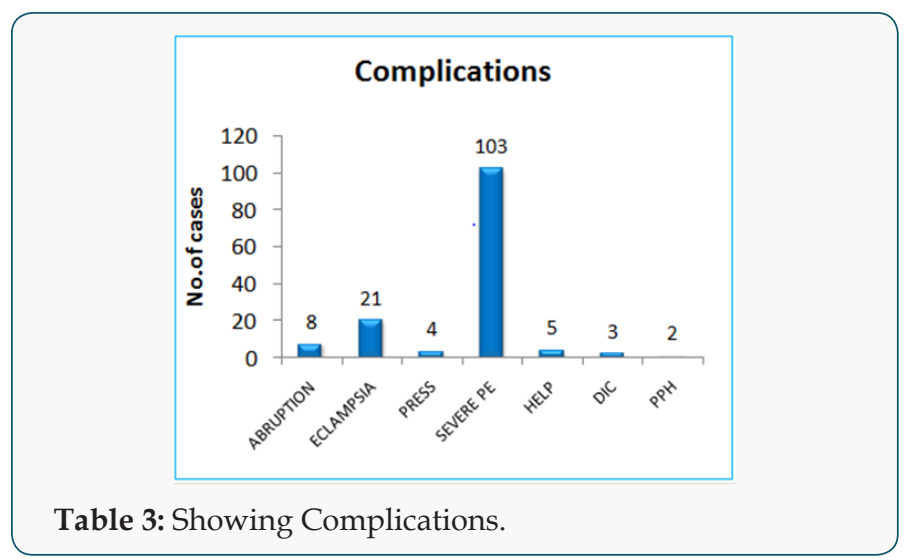

\section{Discussion}

Advanced maternal age has been found to be an independent risk factor for pre-eclampsia, while in this study most of the cases were in the age group 21-25.There is also an increase of incidence in severe pre-eclampsia in unregistered women. This indicates that women who are registered have pre-eclampsia diagnosed earlier and treated [9-11]. We found that anaemia, diabetes mellitus, previous history of pre-eclampsia and multiple pregnancies were associated with pre-eclampsia. Women who have pre-eclampsia in a first pregnancy have seven times the risk of pre-eclampsia in a second pregnancy.58\% of the patients were induced and $45 \%$ of them needed caesarean section due to obstetric indication, the incidence of abruption is more in un booked case. In our study, $10 \%$ of the neonates are with Apgar score less than 5 and 10\% were stillbirths [12,13]. Main factors determining perinatal mortality was the lack of regular antenatal check-ups, complicated cases of pre-eclampsia and lack of awareness regarding significance of symptoms like decreased foetal movements and late arrival at hospital, all contributing to stillbirths. In conclusion, pre-eclampsia tends to threaten maternal health and foetal viability adding to maternal and neonatal mortality and morbidity. There is a high frequency of pre-eclampsia in our setting and the consequences of pre-eclampsia for neonatal mortality and morbidity outcome are alarmingly high [14-16].

\section{Conclusion}

Pre-eclampsia and eclampsia still remains a major problem in developing countries. It is one of the important causes of maternal and perinatal morbidity and mortality probably resulting from inadequate and suboptimal antenatal care and lack of education and awareness amongst people. One maternal death occurs in every 25 eclamptic women [17-20]. A moderate reduction of death of mother and foetus in our institution was possible due to wider use of magnesium sulphate, timed delivery, and proper implementation of emergency obstetric care facilities to mother with eclampsia. The incidence of eclampsia can be reduced by better antenatal care, early recognition and prompt treatment of severe pre-eclampsia. Adequate assessment, investigation, monitoring and intensive care for these women in the antepartum, intrapartum and postpartum period and timely delivery improve significantly the maternal and perinatal outcome. Regular blood pressure monitoring specially in predisposed individuals is essential to detect such high risk cases early and thus prevent complications. This can be done with the help of family physicians.

\section{References}

1. (2012) World Health Organization Fact Sheet.

2. Odegard RA, Vatten LJ, Nilsen ST, Salvesen KA, Austgulen R (2000) Risk factors and clinical manifestations of pre-eclampsia. Br J Obstet Gynaecol 107(11): 1410-1416.

3. Singhal S, Deepika, Anshu, Nanda S (2009) Maternal and perinatal outcome in severe pre-eclampsia and eclampsia. South Asian Federation of Obstetrics and Gynaecology 1(3): 25-28. 
4. Katz VL, Farmer R, Kuller JA (2000) Preeclampsia into eclampsia: toward a new paradigm. Am J Obstet Gynecol 182(6): 1389-1396.

5. Carty DM, Delles C, Dominiczak AF (2010) Preeclampsia and future maternal health. J Hypertens 28(7): 1349-1355.

6. Duley L (2009) The global impact of pre-eclampsia and eclampsia. Semin Perinatol 33(3): 130-137.

7. Sibai B, Dekker G, Kupferminc M (2005) Pre-eclampsia. Lancet 365(9461): 785-799.

8. Tan KH, Kwek K, Yeo GS (2006) Epidemiology of preeclampsia and eclampsia at the KK women's and children's hospital, Singapore. Singapore Med J 47(1): 48-53.

9. Sibai BM (2005) Diagnosis, prevention, and management of eclampsia. Obstet Gynecol 105(2): 402-410.

10. Alvarez Navascués R, Marín R (2001) Severe maternal complications associated with pre-eclampsia: an almost forgotten pathology? Nefrologia 21(6): 565-573.

11. Roberts JM (1998) Endothelial dysfunction in preeclampsia. Semin Reprod Endocrinol 16(1): 5-15.

12. (2009) Multidisciplinary management of severe preeclampsia (PE) experts' guidelines 2008 28: 275-281.

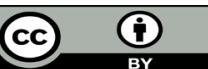

This work is licensed under Creative Commons Attribution 4.0 License

To Submit Your Article Click Here:

Submit Article

DOI: $10.32474 /$ IGWHC.2018.01.000125
13. Pottecher T, Luton D (2009) Multidisciplinary management of preeclampsia. French. Issy Les Moulineaux, Elsevier, Masson SAS, France.

14. Minire A, Mirton M, Imri V, Lauren M, Aferdita M (2013) Maternal complications of preeclampsia. Med Arch 67(5): 339-341.

15. Churchill D, Perry IJ, Beevers DG (1997) Ambulatory blood pressure in pregnancy and foetal growth. Lancet 349(9044): 7-10.

16. Kuklina EV, Ayala C, Callaghan WM (2009) Hypertensive disorders and severe obstetric morbidity in the United States. Obstet Gynecol 113(6): 1299-1306.

17. Duley L (2003) Pre-eclampsia and the hypertensive disorders of pregnancy. British Medical Bulletin 67: 161-176.

18.(2008) Lack of preeclampsia awareness increases risk of infant mortality, press release, preeclampsia foundation.

19. Shaikh S, Ruby AJ, Piotrowski M (2003) Pre eclampsia related chorioretinopathy with Purtscher's like findings and macular ischaemia. Retina 23: 247-250.

20. Naseer D, Ataullah K, Nudrat E (2000) Perinatal and maternal outcome of eclamptic patients admitted in Nishtar Hospital, Multan. J Coll Physician Surg Pak 10: 261-264.

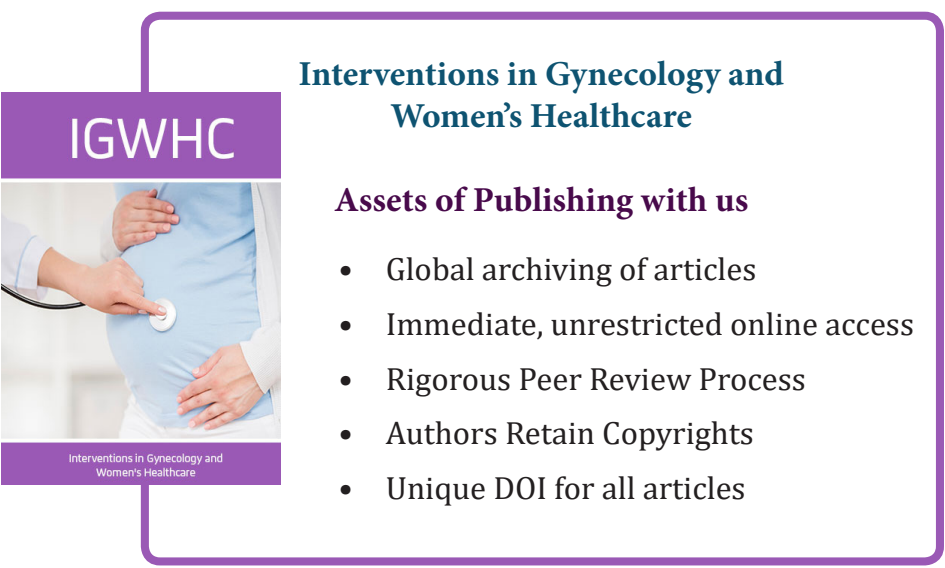

